\title{
ON SMOOTH LOCALLY O-MINIMAL FUNCTIONS
}

\author{
ANDREAS FISCHER
}

\begin{abstract}
We study the $\mathcal{C}^{\infty}$-smooth functions which are locally definable in an o-minimal expansion of the real exponential field with some additional smoothness conditions. Here, the local definability generalizes the subanalytic setting to more transcendental sets and functions. The focus is set on the locally definable diffeomorphisms between manifolds, for which we prove analogies to classical differential geometric results. Moreover, we investigate the relation between classical diffeomorphy and locally definable diffeomorphy.
\end{abstract}

\section{Introduction}

There are two established ways of the locally o-minimal setting. Van den Dries and Miller introduced in [9] the notion of analytic-geometric category as a local version of o-minimal expansions of $\mathbb{R}_{\mathrm{an}}$-the real field with restricted analytic functions, see [5] — and Shiota introduced the notion of $\mathfrak{X}$ set and $\mathfrak{X}$ function in [32] thereby developing a theory of $\mathfrak{X}$ differential geometry. Here, we will follow Shiota's concept. In the following, let $\mathcal{M}$ denote an o-minimal expansion of the real numbers. Here, definable always means definable in $\mathcal{M}$ with parameters in $\mathbb{R}$. A sound geometrical approach to o-minimal structures can be found in [4], [6], [9].

A subset $X$ of $\mathbb{R}^{n}$ is called an $\mathcal{S}$ set if for every $x \in \mathbb{R}^{n}$ there exists an open ball with center $x$ whose intersection with $X$ is definable. A continuous function is called an $\mathcal{S}$ function if its graph is an $\mathcal{S}$ set. If $\mathcal{M}$ is $\mathbb{R}_{\text {an }}$ then the corresponding $\mathcal{S}$ sets and the $\mathcal{S}$ functions are precisely the subanalytic sets and the subanalytic functions. As a generalization of the semialgebraic an the subanalytic setting, Shiota developed the fundamental theory of $\mathfrak{X}$ sets

Received June 2, 2012; received in final form August 15, 2012.

2010 Mathematics Subject Classification. Primary 32B20, 14P10. Secondary 03C64, $26 \mathrm{~B} 05$. 
and $\mathfrak{X}$ functions in [32]. Both the collection of definable sets of an o-minimal structure and the collection of $\mathcal{S}$ sets satisfy the axioms of the $\mathfrak{X}$ notation.

In the present paper, we discuss $\mathcal{C}^{\infty}$-smooth $\mathcal{S}$ functions and $\mathcal{S}$ manifolds for $\mathcal{M}$ being an o-minimal expansion of the real exponential field which additionally admits $\mathcal{C}^{\infty}$-smooth cell decomposition (see Section 2). Our main focus is set on $\mathcal{C}^{\infty}$-smooth manifolds and functions, which have not been considered in [32]. Though the $\mathfrak{X}$ notation is already established, we prefer our notation that we consider to be easier to work with.

In the second section, we briefly recall the basics of o-minimality and the $\mathfrak{X}$ notation.

In the third section, we commence the study of $\mathcal{S}$ functions. There is a crucial difference between o-minimal geometry and $\mathcal{S}$ geometry, namely, the projection of an $\mathcal{S}$ set is not necessarily an $\mathcal{S}$ set. This leads to several algebraic problems, because, in general, sums, products and compositions of $\mathcal{S}$ functions are not $\mathcal{S}$ functions. The largest class of $\mathcal{S}$ functions which is closed under these basic operations are the uniform $\mathcal{S}$ functions; that is, functions which map bounded sets on bounded sets. (These are those functions for which Shiota established the above mentioned $\mathfrak{X}$ differential topology.) Despite their algebraic amenities, the uniform $\mathcal{S}$ functions have some crucial disadvantage. The $\mathcal{S}$ notation is a local o-minimal concept so that for a definable set $A$, one expects that all definable function on $A$ are also locally definable (in an appropriate sense). So we extend our studies to strongly locally definable functions, in short $\mathcal{S}_{\text {sld }}$ functions. This class of functions can be described as the localization of the uniform $\mathcal{S}$ functions by the strictly positive uniform $\mathcal{S}$ functions, and we develop some basic properties of these functions.

The main result of the forth section is the $\mathcal{C}^{\infty}$-smooth approximation of differentiable $\mathcal{S}_{\text {sld }}$ functions with respect to an appropriate topology. This topology allows us to study the $\mathcal{C}^{\infty}$-smooth uniform $\mathcal{S}$ diffeomorphisms. As immediate applications we obtain the existence of $\mathcal{C}^{\infty}$-smooth $\mathcal{S}$ partition of unity and separation of closed $\mathcal{S}$ sets.

In the fifth section, we will study $\mathcal{S}_{\text {sld }}$ diffeomorphisms and their smoothening. There, we also prove that the $\mathcal{C}^{\infty}$-smooth uniform $\mathcal{S}$ functions on a closed $\mathcal{C}^{\infty}$-smooth $\mathcal{S}$ manifold are dense in the continuously differentiable functions with respect to the classical Whitney topology.

The different notions of diffeomorphisms gives rise to consider different concepts of diffeomorphism classes, and we will do so in the sixth section. There we include cardinality questions of diffeomorphism classes of $\mathcal{S}$ manifolds.

To obtain a better understanding of the relation between the set of $\mathcal{S}$ diffeomorphisms and $\mathcal{C}^{m}$ diffeomorphisms, compositions of $\mathcal{S}$ functions must be studied. In the final seventh section, we explore sums and compositions of $\mathcal{S}$ functions. Both operations generate a larger class of functions assigned to the $\mathcal{S}$ functions, namely the locally definable functions. We discuss some 
differential topological properties of these functions and show how to construct them from the $\mathcal{S}_{\text {sld }}$ functions and from the $\mathcal{S}$ functions.

\section{O-minimal structures and the $\mathcal{S}$ notation}

2.1. O-minimal structures. A subset $X$ of $\mathbb{R}^{n}$ is semialgebraic if $X$ is the solution of a finite family of real polynomial inequalities. A function is called semialgebraic if its graph is semialgebraic. A sound introduction to semialgebraic geometry is provided by [3].

Definition 2.1. A structure $\mathcal{M}$ is a collection $\left(M_{n}\right)_{n \in \mathbb{N}}$, where each $M_{n}$ consists of subsets of $\mathbb{R}^{n}$ which are called definable subsets, such that:

(S1) every semialgebraic set is definable,

(S2) finite unions, finite intersections and complements of definable sets are again definable,

(S3) images of definable sets under linear maps are again definable.

The structure $\mathcal{M}$ is called $o$-minimal if in addition

(O) any definable set in $R$ is a finite union of points and open intervals.

A function $f$ is called definable if its graph is definable.

The structure axioms (S1) to (S3) allow us to perform the basic set theoretical operations, addition, multiplication, and they guarantee the definability of the order relation. They additionally imply that compositions of definable functions are again definable, and that images and preimages of definable sets under definable functions are definable. These facts can be found in [4], [6], [9].

In the presence of the axioms $(\mathrm{S} 1)$ to $(\mathrm{S} 3)$, axiom $(\mathrm{O})$ implies what Grothendieck claimed for a category to be tame, see [17], and it provides us with powerful geometric tools. Examples of o-minimal structures are found in [2], [5], [8], [10], [19], [21], [27], [28], [36], [37]. Speissegger proved in [34] that the Pfaffian closure of an o-minimal structure is again o-minimal.

\subsection{Local definability.}

Definition 2.2. Let $\mathcal{M}$ be an o-minimal structure.

(a) A subset $A \subset \mathbb{R}^{n}$ is an $\mathcal{S}$ set if for all $x \in \mathbb{R}^{n}$ there exists a ball $B$ with center $x$ such that $B \cap A$ is definable.

(b) A continuous function $f: A \rightarrow B$ is an $\mathcal{S}$ function if its graph is an $\mathcal{S}$ set.

(c) A manifold $M$ which is imbedded in $\mathbb{R}^{n}$ is called an $\mathcal{S}$ manifold if its underlying set is an $\mathcal{S}$ set.

Similar to structures, finite unions and intersections as well as complements of $\mathcal{S}$ sets are $\mathcal{S}$ sets again. Moreover, if $A$ is a bounded $\mathcal{S}$ set, then $A$ is definable. 
The collection of all $\mathcal{S}$ sets satisfies Shiota's axioms for $\mathfrak{X}$ sets, see [32]. This observation allows us to use many facts which Shiota established in [32, Sections II.1 and II.5].

2.3. Smooth cell decomposition. In our context, the rather technical definition of cell decomposition is not required, and we refer the reader to [9] for details. For us, the following definition is sufficient. Let $m \in \mathbb{N} \cup$ $\{\infty, \omega\}$. A definable function $f: A \rightarrow \mathbb{R}$ is called a $\mathcal{C}^{m}$ function if there exists a definable open neighbourhood $U$ of $A$ and a definable $\mathcal{C}^{m}$ function $F: U \rightarrow \mathbb{R}$ such that $\left.F\right|_{A}=f$. Recall that an o-minimal structure admits $\mathcal{C}^{\infty}$-smooth cell decomposition, if for every definable function $f: \mathbb{R}^{n} \rightarrow \mathbb{R}$ there exists a finite partition of $\mathbb{R}^{n}$ into definable sets $A_{1}, \ldots, A_{k}$ such that $f$ restricted to $A_{i}$ is a $\mathcal{C}^{m}$ function.

Every o-minimal structure admits $\mathcal{C}^{m}$ cell decomposition for finite $m$, see [6, Section 7.3]. By applying $\mathcal{C}^{m}$ cell decomposition to a definable unary function, we see that every definable unary function is piecewise $\mathcal{C}^{m}$. Recently, LeGal and Rolin constructed in [21] an o-minimal structure that does not admit $\mathcal{C}^{\infty}$ cell decomposition. However, most known o-minimal expansions of the real numbers admit at least $\mathcal{C}^{\infty}$ cell decomposition, many of them even analytic cell decomposition. The Pfaffian closure of an o-minimal structure preserves analytic cell decomposition, cf. [22]. Both $\mathbb{R}_{\text {an }}$ and $\mathbb{R}_{\exp }$ admit analytic cell decomposition and so do all the substructures of their Pfaffian closures. The o-minimal expansions constructed in [8], [10], [19] admit analytic cell decomposition. The o-minimal structures generated by certain quasi-analytic Denjoy Carleman classes and expanded by the exponential function admit $\mathcal{C}^{\infty}$-smooth cell decomposition but not analytic cell decomposition, cf. [28].

\subsection{Differential-topology.}

Convention: All manifolds are assumed to be imbedded into some $\mathbb{R}^{n}$.

For every $\mathcal{C}^{1}$ manifold $M$, we denote by $T_{x} M$ the tangent space in $M$ at $x$. By abuse of notation we denote a topological manifold by $\mathcal{C}^{0}$ manifold.

Let $M \subset \mathbb{R}^{n}$ be a $\mathcal{C}^{0}$ manifold and let $\varepsilon: M \rightarrow(0, \infty)$ be a continuous map. Then we set $D^{0}(f-g):=f-g$. Let $m \geq 1$ be any integer. Suppose that $M \subset \mathbb{R}^{n}$ and $N \subset \mathbb{R}^{k}$ are $\mathcal{C}^{m}$ manifolds, and that $f: M \rightarrow N$ is some $\mathcal{C}^{m}$ function. For $x \in M$, let $d f_{x}: T_{x} M \rightarrow \mathbb{R}^{k}$ denote the differential of $f$ at $x$. Extend $d f_{x}$ to a map from $\mathbb{R}^{n}$ to $\mathbb{R}^{k}$ by composing it with the orthogonal projection $\mathbb{R}^{n} \rightarrow T_{x} M$ and denote the resulting map from $M$ to $\mathbb{R}^{n k}$ by $D f$. Then, the map $(f, D f): M \rightarrow \mathbb{R}^{k} \times \mathbb{R}^{n k}$ is a $\mathcal{C}^{m-1}$ map. For $\ell=1, \ldots, m$ we inductively define the $\mathcal{C}^{m-\ell}$-map $D^{\ell} f: M \rightarrow \mathbb{R}^{n^{\ell} k}$ by $D^{\ell} f:=D\left(D^{\ell-1} f\right)$.

To simplify notation, we write

$$
\|f-g\|_{m}:=\sum_{\ell=0}^{m}\left\|D^{\ell}(f-g)\right\| .
$$


For a continuous function $\varepsilon: M \rightarrow(0, \infty)$ let

$$
U_{\varepsilon}^{m}(f):=\left\{g \in \mathcal{C}^{m}(M, N) ;\|f-g\|_{m}<\varepsilon \text { on } M\right\} .
$$

For $m \geq 0$, the $\mathcal{C}^{m}$ topology on $\mathcal{C}^{m}(M, N)$ is the coarsest topology for which the above sets $U_{\varepsilon}^{m}(f)$ are open, where $f$ ranges over all elements of $\mathcal{C}^{m}(M, N)$, and $\varepsilon$ over all elements of $\mathcal{C}^{0}(M,(0, \infty))$.

To obtain the o-minimal version of the Whitney topology, we first note that the tangent mapping of a definable $\mathcal{C}^{1}$ manifold is definable. So let $M \subset \mathbb{R}^{n}$ and $N \subset \mathbb{R}^{m}$ be definable $\mathcal{C}^{m}$ manifolds, and let $\mathcal{C}_{\text {def }}^{m}(M, N)$ denote the definable $\mathcal{C}^{m}$ functions from $M$ to $N$. Set

$$
U_{\varepsilon, \mathrm{def}}^{m}(f):=\left\{g \in \mathcal{C}_{\mathrm{def}}^{m}(M, N) ;\|f-g\|_{m}<\varepsilon \text { on } M\right\} .
$$

Then the $\mathcal{C}_{\text {def }}^{m}$ topology on $\mathcal{C}_{\text {def }}^{m}(M, N)$ is the coarsest topology for which the above sets $U_{\varepsilon \text {,def }}^{m}(f)$ are open, where $f \in \mathcal{C}_{\text {def }}^{m}(M, N)$ and $\varepsilon \in \mathcal{C}_{\text {def }}^{0}(M,(0, \infty))$.

Analogously we obtain the $\mathcal{S}$ version of the Whitney topology. Let $M \subset \mathbb{R}^{n}$ and $N \subset \mathbb{R}^{k}$ be imbedded $\mathcal{S}^{m}$ manifolds, and let $\mathcal{S}^{m}(M, N)$ denote the $\mathcal{C}^{m} \mathcal{S}$ functions from $M$ to $N$. Set

$$
U_{\varepsilon, \mathcal{S}}^{m}(f):=\left\{g \in \mathcal{S}^{m}(M, N) ;\|f-g\|_{m}<\varepsilon \text { on } M\right\} .
$$

Then the $\mathcal{S}^{m}$ topology on $\mathcal{S}^{m}(M, N)$ is the coarsest topology for which the above sets $U_{\varepsilon, \mathcal{S}}^{m}(f)$ are open, where $f \in \mathcal{S}^{m}(M, N)$ and $\varepsilon: M \rightarrow(0, \infty)$ is a continuous $\mathcal{S}$ function.

Similar to the Whitney topology, the definable $\mathcal{C}^{m}$ diffeomorphisms between two definable $\mathcal{C}^{m}$ submanifolds $M$ and $N$ form an open subset of $\mathcal{C}_{\text {def }}^{m}(M, N)$ with respect to the $\mathcal{C}_{\text {def }}^{m}$-topology, cf. [29, Lemma II.1.7].

There exist several versions of the concept of closed manifold. Here, a submanifold $M^{\prime}$ of $M$ is called closed if the underlying set of $M^{\prime}$ is closed in $M$. If $M$ is not given, then the underlying set of $M^{\prime}$ is assumed to be closed in some $\mathbb{R}^{n}$.

\section{Strongly locally definable functions}

3.1. Basic properties. In this introducing section, we fix an arbitrary ominimal structure and its corresponding collection of $\mathcal{S}$ sets.

Let $U \subset \mathbb{R}^{n}$. An $\mathcal{S}$ function $f: U \rightarrow \mathbb{R}^{m}$ is called uniform, if $f$ maps bounded subsets of $U$ to bounded subsets of $\mathbb{R}^{n}$. Within the class of uniform $\mathcal{S}$ functions we can perform the usual operations such as sum, product and composition. The set of uniform $\mathcal{C}^{m} \mathcal{S}$ functions is abbreviated by $\mathcal{S}_{u}^{m}$ functions. Notice that linear projections, polynomials, Lipschitz continuous $\mathcal{S}$ functions and $\mathcal{S}$ functions with closed domain are uniform. This leads to a category which we call the $\mathcal{S}$-category. Its objects are the $\mathcal{S}$ sets and its morphisms are the uniform $\mathcal{S}$ functions.

If $U \subset \mathbb{R}^{n}$ is a open definable proper subset, then not every definable continuous function is an $\mathcal{S}_{u}$ function. Moreover, the reciprocal of a strictly 
positive $\mathcal{S}_{u}$ function is not necessarily an $\mathcal{S}_{u}$ function. This motivates us to study strongly locally definable functions.

Definition 3.1. Let $U \subset \mathbb{R}^{n}$ be an $\mathcal{S}$ set. A function $f: U \rightarrow \mathbb{R}^{m}$ is called strongly locally definable, in short an $\mathcal{S}_{\text {sld }}$ function, if for every $x \in \mathbb{R}^{n}$ there is an open ball $B$ with center $x$ such that $f$ restricted to $U \cap B$ is definable.

Evidently, the graph of an $\mathcal{S}_{\text {sld }}$ function is an $\mathcal{S}$ set so that every continuous $\mathcal{S}_{\text {sld }}$ function is an $\mathcal{S}$ function. Sum and product of $\mathcal{S}_{\text {sld }}$ functions are $\mathcal{S}_{\text {sld }}$ functions. Moreover, the partial derivatives of differentiable strongly locally definable function are strongly locally definable functions. The $\mathcal{C}^{k}$ singular points ( $k$ finite) of a continuous strongly locally definable function is an $\mathcal{S}$ set. We abbreviate strongly locally definable $\mathcal{C}^{m}$ function by $\mathcal{S}_{\text {sld }}^{m}$ function for $m \in \mathbb{N} \cup\{\infty, \omega\}$.

In general, the $\mathcal{S}_{\text {sld }}$ functions are not closed under composition. However, we have the following proposition.

Proposition 3.2. Let $f: U \rightarrow V$ be an $\mathcal{S}_{\text {sld }}$ function, let $g: W \rightarrow U$ be an $\mathcal{S}_{u}$ function, and let $h: V \rightarrow X$ be definable. Then $f \circ g$ and $h \circ f$ are $\mathcal{S}_{\text {sld }}$ functions.

Proof. Suppose that $V \subset \mathbb{R}^{m}$. Let $x \in \mathbb{R}^{m}$ and let $B$ be some open ball with center $x$. Then $g: V \cap B \rightarrow U$ is definable. Moreover, the image of $V \cap B$ under $g$ is a bounded definable set. Hence, the function $f \circ g$ restricted to $B \cap V$ is definable, so that $f \circ g$ is strongly locally definable.

Suppose that $U \subset \mathbb{R}^{n}$. Let $x \in \mathbb{R}^{n}$. Then there is a ball $B$ with center $x$ such that $f$ restricted to $B \cap U$ is definable. Hence, $h \circ f$ restricted to $B \cap U$ is definable.

The $\mathcal{S}_{\text {sld }}^{m}$ functions can be constructed from the $\mathcal{S}_{u}$ functions as follows.

Proposition 3.3. Let $m \in \mathbb{N} \cup\{\infty, \omega\}$. Let $U \subset \mathbb{R}^{n}$ be an open $\mathcal{S}$ set, and let $W=\mathcal{S}^{m}(U,(0,1))$. Then

$$
\mathcal{S}_{\text {sld }}^{m}(U, \mathbb{R})=W^{-1} \mathcal{S}_{u}^{m}(U, \mathbb{R})
$$

Proof. Let $f \in \mathcal{S}_{\text {sld }}^{m}(U, \mathbb{R})$. Then $g:=1 /\left(1+f^{2}\right)$ is a strictly positive bounded $\mathcal{S}^{m}$ function, and $f g$ is a uniform $\mathcal{S}^{m}$ function. So $f=(1 / g) f g \in$ $W^{-1} \mathcal{S}_{u}^{m}(U, \mathbb{R})$. tion.

Alternatively, the $\mathcal{S}_{\text {sld }}^{m}$ functions satisfies the following uniqueness condi-

Proposition 3.4. Let $m \in \mathbb{N} \cup\{\infty, \omega\}$. Let $U \subset \mathbb{R}^{n}$ be an open $\mathcal{S}$ set. Then the $\mathcal{S}_{\text {sld }}^{m}$ functions from $U$ to $\mathbb{R}$ is the only ring $\mathcal{A}$ which satisfies:

(a) $\mathcal{S}_{u}^{m}(U, \mathbb{R}) \subset \mathcal{A}$,

(b) $\mathcal{A} \subset \mathcal{S}^{m}(U, \mathbb{R})$,

(c) if $f \in \mathcal{A}$ with $f>0$ on $U$, then $1 / f \in \mathcal{A}$. 
Proof. By Proposition 3.3, the smallest algebra $\mathcal{A}$ satisfying the above axioms is $\mathcal{S}_{\text {sld }}^{m}(U, \mathbb{R})$. Let $\mathcal{A}$ be the largest among the algebras satisfying the above axioms. Let $f \in \mathcal{A}$, then $1+f^{2} \in \mathcal{A}$, thus $1 /\left(1+f^{2}\right) \in \mathcal{A}$. This function is a bounded strictly positive $\mathcal{S}$ function, so it is uniform. Hence, the function $1+f^{2}$ is strongly locally definable, and so is $f^{2}$. Using the identity

$$
f=\frac{1}{4}\left((f+1)^{2}-(f-1)^{2}\right)
$$

we see that $f$ is an $\mathcal{S}_{\text {sld }}^{m}$ function.

\section{Approximation of strongly locally definable functions}

From now on, we fix an o-minimal expansion $\mathcal{M}$ of $\mathbb{R}_{\exp }$ that admits $\mathcal{C}^{\infty}$ smooth cell decomposition.

4.1. Approximation. We shall prove the following approximation theorem.

TheOREM 4.1. Let $U \subset \mathbb{R}^{n}$ be an open $\mathcal{S}$ set, and let $f: U \rightarrow \mathbb{R}$ be an $\mathcal{S}_{\text {sld }}^{m}$ function. For every positive continuous $\mathcal{S}$ function $\varepsilon: U \rightarrow(0, \infty)$ there exists a $\mathcal{C}^{\infty}$-smooth $\mathcal{S}_{\text {sld }}$ function $g: U \rightarrow \mathbb{R}$ such that

$$
\|f-g\|_{m}<\varepsilon
$$

on $U$.

In addition, if $S$ is an $\mathcal{S}$ set closed in $U$ containing the closure of all non$\mathcal{C}^{\infty}$-smooth points of $f$, and if $V$ is an open $\mathcal{S}$ set containing $S$, then we may assume that $f=g$ outside of $V$.

Proof. For every $a \in \mathbb{R}^{n}$ there exists an open ball $B(a)$ with center $a$ such that $f$ restricted to $B(a) \cap U$ is definable. Select a locally finite collection of these balls, $\left\{B_{i} ; i \in \mathbb{N}\right\}$ say, such that $\bigcup_{i \in \mathbb{N}} B_{i}=\mathbb{R}^{n}$. We construct a sequence $\left(f_{i}\right)_{i \in \mathbb{N}}: U \rightarrow \mathbb{R}$ of $\mathcal{S}_{\text {sld }}^{m}$ functions such that the following holds:

(a) the function $f_{i}$ restricted to $U \cap \bigcup_{j \leq i} B_{i}$ is $\mathcal{C}^{\infty}$-smooth,

(b) $f_{i}=f$ outside of $\bigcup_{j<i} B_{i}$,

(c) $f_{i}=f_{i-1}$ outside of $B_{i}$ for $i \geq 1$,

(d) $f_{i}=f$ outside of $V$,

(e) and $\left|f_{i}-f\right|_{m}<\left(1-2^{-i}\right) \varepsilon / 2$ on $U$.

Suppose, for instance, we have already constructed this sequence. Then we may set $g:=\lim _{i \rightarrow \infty} f_{i}$, since $g$ is then a $\mathcal{C}^{\infty}$-smooth function satisfying inequality (2), and $g$ is an $\mathcal{S}$ function because of (d) and because the collection of the balls $B_{i}$ is locally finite.

Set $B_{0}=\varnothing$ and let $f_{0}:=f$. Suppose now that $f_{j}$ is already constructed for $j \leq i-1$. Notice that $f_{i-1}$ restricted to $B_{i}$ is a definable $\mathcal{C}^{m}$ function. So the set of non- $\mathcal{C}^{\infty}$-smooth points is contained in a definable subset $A$ of $B_{i} \cap U \cap V$, closed in $U \cap B_{i}$, of dimension less than $n$. Moreover, because of 
item (a), the function $f_{i}$ restricted to $B_{i} \cap \bigcup_{j<i} B_{j}$ is $\mathcal{C}^{\infty}$-smooth, so that we may assume that $A \cap B_{j}=\varnothing$ for $j<i$. Let $\delta: B_{i} \cap U \rightarrow(0,1]$ be defined by

$$
\delta(u):=\min \left(2^{-i-1} \varepsilon(u), \operatorname{dist}\left(u, \partial\left(U \cap B_{i}\right)\right)\right) .
$$

Because of item (a), there is a definable open set $W \subset B_{i} \cap U$ containing $\bar{A} \cap U$ such that

$$
W \cap\left(\partial\left(U \cap B_{i}\right) \cap \bigcup_{j<i} B_{j}\right)=\varnothing .
$$

By $\left[14\right.$, Theorem 1.1] there is a definable $\mathcal{C}^{\infty}$-smooth function $h: B_{i} \cap U \rightarrow \mathbb{R}$ such that $\left\|h-f_{i-1}\right\|_{m}<\delta$ on $B_{i} \cap U$, and $h=f_{i-1}$ outside of $W$.

Let $f_{i}: U \rightarrow \mathbb{R}$ be defined by

$$
f_{i}(u):= \begin{cases}h(u), & \text { if } u \in B_{i}, \\ f_{i-1}(u), & \text { if } u \notin B_{i} .\end{cases}
$$

Then, by construction, item (e) is satisfied, and $f_{i}$ is a $\mathcal{S}_{\text {sld }}^{m}$ function on $U$. Since $h=f_{i-1}$ outside of $W$, item (d) is satisfied, and (b) and (c) are evidently satisfied by construction. Finally, the $f_{i}$ is $\mathcal{C}^{\infty}$-smooth in $U \cap \bigcup_{j \leq i} B_{j}$.

In general, a close approximation of an $\mathcal{S}_{\text {sld }}$ function by an $\mathcal{S}$ functions is not necessarily an $\mathcal{S}_{\text {sld }}$ function. But if $f$ is an $\mathcal{S}_{u}$ function, every sufficiently close $\mathcal{S}$ function is an $\mathcal{S}_{u}$ function. Hence, we have the following corollary.

COROLlary 4.2. Let $U$ and $V$ be as in the previous theorem, and suppose that $f$ is additionally uniform, then we may assume that $g$ is uniform as well.

4.2. Separation of $\mathcal{S}$ sets. The Euclidean distance function $\operatorname{dist}(-,-)$ is an $\mathcal{S}_{u}$ function if we allow only $\mathcal{S}$ sets as second argument, see [32, II.1.2].

Corollary 4.3. Let $M \subset \mathbb{R}^{n}$ be a $\mathcal{C}^{\infty}$-smooth $\mathcal{S}$ manifold, and let $A, B \subset$ $M$ be disjoint and closed $\mathcal{S}$ sets in $M$. Then there is an $\mathcal{S}_{u}^{\infty}$ function $\phi: M \rightarrow$ $[0, \infty)$ such that $A \subset\{\phi=0\}$ and $B \subset\{\phi=1\}$.

Proof. The sets $A, B$ are closed in $M$. Hence, they are closed in $U:=$ $\mathbb{R}^{n} \backslash \partial M$. Define the open neighborhood $A^{\prime}$ of $A$ by $A^{\prime}:=\{u \in U: \operatorname{dist}(u, A)<$ $\left.\frac{1}{4} \operatorname{dist}(u, B)\right\}$ and $B^{\prime}:=\left\{u \in U: \operatorname{dist}(u, B)<\frac{1}{4} \operatorname{dist}(u, A)\right\}$. Then $A^{\prime}$ and $B^{\prime}$ are disjoint $\mathcal{S}$ sets which are closed in $U$. Let $\phi: U \rightarrow[0,1]$ be defined by $\psi(u)=\min \left(\operatorname{dist}\left(u, A^{\prime}\right), 1\right)$. The set of non- $\mathcal{C}^{\infty}$-smooth points of $\psi$ is contained in an open $\mathcal{S}$ set $V \subset U$ whose closure in $U$ is disjoint from $A \cup B$. Hence, by Theorem 4.1, there is a $\mathcal{C}^{\infty}$-smooth $\mathcal{S}$ function $\phi: U \rightarrow[0, \infty)$ approximating $\psi$ sufficiently good so that $A \subset\{\phi=0\}$ and $B \subset\{\phi=1\}$.

The previous corollary leads to the following weak extension of $\mathcal{C}^{\infty}$-smooth functions. 
Corollary 4.4. Let $M \subset \mathbb{R}^{n}$ be a $\mathcal{C}^{\infty}$-smooth $\mathcal{S}$ manifold, and $A \subset M$ an $\mathcal{S}$ set which is closed in $M$. Let $U \subset M$ be an $\mathcal{S}$ set which is open in $M$ and which contains $A$. If $f: U \rightarrow \mathbb{R}$ is an $\mathcal{S}_{\text {sld }}^{\infty}$ function, then there is an $\mathcal{S}_{\text {sld }}^{\infty}$ function $F: M \rightarrow \mathbb{R}$ with $\left.F\right|_{A}=f \mid A$. If $f$ is uniform we may assume that $F$ is uniform.

Proof. Set $B=M \backslash U$. Select, by Corollary 4.3, an $\mathcal{S}_{u}^{\infty}$ function $\phi: M \rightarrow$ $[0, \infty)$ which vanishes in $B$ and equals 1 in $A$. Define $F: M \rightarrow \mathbb{R}$ by

$$
F(x):= \begin{cases}f(x) \phi(x), & \text { if } x \in U, \\ 0, & \text { if } x \in M \backslash U .\end{cases}
$$

Then $F$ satisfies the conclusion of the corollary. Evidently, if $f$ is uniform, then $F$ is uniform.

4.3. Partition of unity. As next application, we prove a $\mathcal{C}^{\infty}$-smooth partition of unity for $\mathcal{S}$ functions. This concept requires a more restrictive notation of locally finite open cover, otherwise our constructions leave the $\mathcal{S}$ setting.

Definition 4.5. Let $M \subset \mathbb{R}^{n}$ be an $\mathcal{S}$ manifold. An open $\mathcal{S}$-cover of $M$ is a cover $\left\{U_{i} ; i \in \mathbb{N}\right\}$ of $M$ such that:

(a) every $U_{i}$ is an $\mathcal{S}$ set open in $M$,

(b) for every $x \in \mathbb{R}^{n}$ there is a ball $B$ with center $x$ such that there are only

finitely many of the $U_{i}$ which have nonempty intersection with $B$.

While item (a) of the previous definition is quite natural, we shall notice that item (b) implies that every $\mathcal{S}$-cover is a locally finite cover, but item (b) cannot be replaced by locally finite cover.

Example 4.6. Let $U=(0,1)$, and for $i \in \mathbb{N}$, let $U_{i}:=(1 /(i+2), 1 / i)$. Then the collection of the sets $U_{i}$ is a locally finite cover consisting of open $\mathcal{S}$ sets. Let $\rho: \mathbb{R} \rightarrow[0,1]$ be defined by

$$
\rho(x):= \begin{cases}\min (x, 1-x), & \text { if } 0<x<1, \\ 0, & \text { otherwise. }\end{cases}
$$

Let $f:(0,1) \rightarrow \mathbb{R}$ be given by

$$
f(x)=\sum_{i=1}^{\infty} \rho(2 i(i+2) x-1 /(i+2)) .
$$

Then the functions $f_{i}:=\left.f\right|_{U_{i}}$ glue together to $f$ and each of the $f_{i}$ is a continuous $\mathcal{S}$ function. However, the function $f$ is not an $\mathcal{S}$ function. Notice that the cover consisting of the $U_{i}$ fails to be an $\mathcal{S}$-cover at the origin.

However, $\mathcal{C}^{\infty}$-smooth partition of unity for $\mathcal{S}$ functions holds when the locally finite cover is also an $\mathcal{S}$-cover. 
Proposition 4.7. Let $M \subset \mathbb{R}^{n}$ be an $\mathcal{S}^{\infty}$ manifold, and let $\left\{U_{i} ; i \in \mathbb{N}\right\}$ be an $\mathcal{S}$-cover of $M$. Then there are $\mathcal{S}_{u}^{\infty}$ functions $\phi_{i}: M \rightarrow[0,1]$ such that:

(a) $\sum_{i=1}^{\infty} \phi_{i}=1$,

(b) $\operatorname{supp}\left(\phi_{i}\right) \subset U_{i}$.

Proof. For every $i \in \mathbb{N}$, let

$$
V_{i}:=\left\{x \in U_{i} ; \operatorname{dist}\left(x, U_{i} \backslash \bigcup_{j \neq i} U_{j}\right) \leq 3 \operatorname{dist}\left(x, M \backslash U_{i}\right)\right\}
$$

and

$$
W_{i}:=\left\{x \in M ; \operatorname{dist}\left(x, U_{i} \backslash \bigcup_{j \neq i} U_{j}\right) \geq 6 \operatorname{dist}\left(x, M \backslash U_{i}\right)\right\} .
$$

Then $V_{i}$ and $W_{i}$ are $\mathcal{S}$-subsets of $M$, closed in $M$, and $W_{i} \cap V_{i}=\varnothing$ for $I \in \mathbb{N}$. Moreover, the $V_{i}$ cover $M$. Select by Corollary 4.3 an $\mathcal{S}_{u}^{\infty}$ function $\psi_{i}: M \rightarrow[0, \infty)$ which vanishes on $W_{i}$ and equals 1 in $V_{i}$. Then the functions

$$
\phi_{i}=\frac{\psi_{i}}{\sum_{j=1}^{\infty} \psi_{j}}
$$

form an $\mathcal{S}_{u}^{\infty}$-partition of unity, and $\operatorname{supp}\left(\phi_{i}\right) \subset U_{i}$.

As a consequence, we obtain the following gluing property for $\mathcal{S}_{\text {sld }}$ functions.

Corollary 4.8. Let $M \subset \mathbb{R}^{n}$ be an $\mathcal{S}^{\infty}$ manifold, let $\left\{U_{i} ; i \in \mathbb{N}\right\}$ be an $\mathcal{S}$-cover of $M$, and let $f_{i}: U_{i} \rightarrow \mathbb{R}$ be $\mathcal{S}_{\text {sld }}^{m}$ functions for $i \in \mathbb{N}$. Then, for any subset $S$ of $\mathbb{N}$, the function

$$
f=\sum_{i \in S} \phi_{i} f_{i}
$$

is an $\mathcal{S}_{\text {sld }}^{m}$ function, where the $\phi_{i}$ are taken from Proposition 4.7 .

Proof. For every $i$, the product $\phi_{i} f_{i}$ is an $\mathcal{S}_{\text {sld }}^{m}$ function whose support is contained in $M \cap \operatorname{supp} \phi_{i}$. Thus, for every $x \in \mathbb{R}^{n}$ there is a ball $B$ with center $x$ such that $B$ intersects only with finitely many of the supports of $\phi_{i} f_{i}$. Hence, the function $f$ restricted to $B \cap M$ is definable, so $f$ is an $\mathcal{S}_{\text {sld }}$ function.

\section{Diffeomorphisms}

In literature, see [32], the study of $\mathcal{S}^{m}$ diffeomorphisms is reduced to those for which either $f$ or $f^{-1}$ is a uniform function. One essential reason is that differential topological methods require classes of functions which are closed under compositions, and the uniform $\mathcal{S}$ functions are the largest class of $\mathcal{S}$ functions which are closed under composition. 
5.1. Diffeomorphisms and uniform functions. So we commence the study of $\mathcal{S}$ diffeomorphisms with the uniform diffeomorphisms. We fix a strictly positive integer $m$. To avoid confusions, we agree to the following denomination.

(a) A diffeomorphism $f: M \rightarrow N$ is an $\mathcal{S}_{u}^{m}$ diffeomorphism or a uniform $\mathcal{S}^{m}$ diffeomorphism, if $f$ is additionally an $\mathcal{S}_{u}^{m}$ function.

(b) If both $f$ and $f^{-1}$ are $\mathcal{S}_{u}^{m}$ diffeomorphisms, we say that $f$ is a bi-uniform $\mathcal{S}^{m}$ diffeomorphism.

In [32], Shiota has developed the following differential topological results about uniform $\mathcal{S}$ diffeomorphisms.

THEOREM 5.1.

(a) The set of $\mathcal{S}_{u}^{1}$ diffeomorphisms between two $\mathcal{S}^{1}$ manifolds $M \subset \mathbb{R}^{n}$ and $N \subset \mathbb{R}^{m}$ is open in $\mathcal{S}_{u}^{1}(M, N)$.

(b) The set $\mathcal{S}_{u}^{m}(M, N)$ is dense in $\mathcal{S}_{u}^{1}(M, N)$ for $m<\infty$.

(c) If $f: M \rightarrow N$ is a bi-uniform $\mathcal{S}^{m}$ diffeomorphism and $g$ is sufficiently close to $f$ (with respect to the $\mathcal{S}^{m}$-topology), then $g^{-1}$ is close to $f^{-1}$ for $0<m<\infty$.

Proof. See [32, Theorem II.5.2 and Lemma II.5.3]

If $M^{\prime}$ is a closed $\mathcal{S}^{m}$ manifold of the $\mathcal{S}^{m}$ manifold $M$, then the restriction map

$$
\text { res }: \mathcal{S}_{u}^{m}(M, \mathbb{R}) \rightarrow \mathcal{S}_{u}^{m}\left(M^{\prime}, \mathbb{R}\right)
$$

is continuous. For further $\mathcal{S}^{m}$ manifolds $N$ and $P$, and an $\mathcal{S}_{u}^{m}$-mapping $h: N \rightarrow P$, the induced mapping $h_{*}: \mathcal{S}_{u}^{m}(M, N) \rightarrow \mathcal{S}_{u}^{m}(M, P)$, defined by

$$
h_{*}(f):=h \circ f
$$

is also continuous. Moreover, every $\mathcal{S}^{\infty}$ manifold admits an $\mathcal{S}_{u}^{\infty}$ retraction $\rho: U \rightarrow M$; that is, a uniform $\mathcal{S}^{\infty}$ function $\rho: U \rightarrow M$, where $U$ is an open neighbourhood of $M$ such that $\rho=$ id on $M$. The proof works as for $\mathcal{S}^{m}$ manifolds, cf. [32, II.5]. In some special cases, we can still use differential topological methods to obtain density results for $\mathcal{S}_{\text {sld }}$ functions. We note the following corollary of Theorem 4.1.

COROLlaRY 5.2. Let $m$ be a positive integer. Let $M \subset \mathbb{R}^{n}$ be an $\mathcal{S}^{\infty}$ manifold. Then $\mathcal{S}_{\text {sld }}^{\infty}(M, \mathbb{R})$ is dense in $\mathcal{S}_{\text {sld }}^{m}(M, \mathbb{R})$.

Proof. Let $\varepsilon: M \rightarrow(0, \infty)$ a continuous $\mathcal{S}_{u}$ function, and let $f \in \mathcal{S}_{\text {sld }}^{m}(M, \mathbb{R})$. Select an $\mathcal{S}_{u}^{\infty}$ retraction $\rho: U \rightarrow M$ of $M$. Then, by Proposition 3.2, the function $F:=f \circ \rho: U \rightarrow \mathbb{R}$ is an $\mathcal{S}_{\text {sld }}^{m}$ extension of $f$. Let $\tilde{\varepsilon}:=\varepsilon \circ \rho$. By Corollary 4.2 , there is a $G \in \mathcal{S}_{\text {sld }}^{\infty}(U, \mathbb{R})$ with $\|G-F\|_{m}<\tilde{\varepsilon}$ on $U$. Hence, if $g$ is the restriction of $G$ to $M$, the continuity of the restriction mapping res implies that $g$ is close to $f$. 
For the following $\mathcal{C}^{\infty}$-smooth version of Theorem 5.1(b), we have to restrict ourselves to uniform functions.

Corollary 5.3. Let $M \subset \mathbb{R}^{n}$ and $N \subset \mathbb{R}^{p}$ be $\mathcal{S}^{\infty}$ manifolds. Then $\mathcal{S}_{u}^{\infty}(M$, $N)$ is dense in $\mathcal{S}_{u}^{m}(M, N)$ with respect to the $\mathcal{S}^{m}$-topology.

Proof. Let $f: M \rightarrow N$ be a definable $\mathcal{S}_{u}^{m}$ function. Select an $\mathcal{S}_{u}^{\infty}$ retraction $\rho: U \rightarrow N$ where $U$ is an open $\mathcal{S}$ neighborhood of $N$ in $\mathbb{R}^{n}$. The mapping $\rho_{*}: \mathcal{S}_{u}^{m}(M, U) \rightarrow \mathcal{S}_{u}^{m}(M, N)$ is continuous. Take a sufficiently close $\mathcal{S}_{u}^{\infty}$ approximation $g: M \rightarrow U$ of $f$, and set $h:=r_{*}(g)$. Note that $h$ is $\mathcal{C}^{\infty}$-smooth, and that $h$ is close to $f$.

Hence we obtain the following statement.

TheOREm 5.4. Let $m \geq 1$ be an integer. Two $\mathcal{S}^{\infty}$ manifolds $M \subset \mathbb{R}^{n}$ and $N \subset R^{m}$ are $\mathcal{S}_{u}^{\infty}$-diffeomorphic if and only if they are $\mathcal{S}_{u}^{m}$-diffeomorphic.

Proof. By Theorem 5.1(b), the $\mathcal{S}_{u}^{m}$ diffeomorphisms from $M$ to $N$ are an open subset of $\mathcal{S}_{u}^{m}(M, N)$. Hence, it is enough to choose an $\mathcal{S}_{u}^{\infty}$ function $\phi: M \rightarrow N$ close enough to a given $\mathcal{S}_{u}^{m}$ diffeomorphism $f: M \rightarrow N$. Such $\phi$ exists by Lemma 5.3.

5.2. Strongly locally definable diffeomorphisms. We shall now prove a generalisation of Theorem 5.4 to $\mathcal{S}_{\text {sld }}^{m}$ functions without using compositions of functions.

TheOREM 5.5. Let $m \geq 1$. Let $M$ and $N$ be $\mathcal{S}^{\infty}$ manifolds. Every $\mathcal{S}_{\text {sld }}^{m}$ diffeomorphism $f: M \rightarrow N$ can be closely approximated by an $\mathcal{S}_{\text {sld }}^{\infty}$ diffeomorphism $g: M \rightarrow N$.

Proof. Let $\varepsilon: M \rightarrow(0, \infty)$ be an $\mathcal{S}_{\text {sld }}^{0}$ function. We construct an $\mathcal{S}_{\text {sld }}^{\infty}$ diffeomorphism $g: M \rightarrow N$ with $\|g-f\|_{m}<\varepsilon$ on $M$.

Select for every $i \in \mathbb{N}$ a definable continuous function $\varepsilon_{i}: M \cap B(i-1, i+$ $1) \rightarrow(0,1)$ such that $\varepsilon_{i}<\varepsilon / 3$ on $M \cap B(i-1, i+1)$ and such that $\varepsilon_{i}(x) \rightarrow$ 0 as $x \rightarrow \partial(M \cap B(i-1, i+1))$.

Let $g_{-1}:=f$. We construct a sequence of $\mathcal{S}_{\text {sld }}^{m}$ diffeomorphisms $g_{i}: M \rightarrow N$, $i \in \mathbb{N}$, such that:

(a) $\left\|g_{i}-f\right\|_{m}<\varepsilon$ on $M$

(b) $g_{i}=g_{i-1}$ outside of $M \cap B(i-3 / 4, i+1)$,

(c) $g_{i}$ is $\mathcal{C}^{\infty}$-smooth in $M \cap B_{i+1}(0)$,

(d) $\left\|g_{i}-g_{i-1}\right\|_{m}<\varepsilon_{i}$ on $M \cap B(i-1, i+1)$

for $i \geq 0$. By [14, Theorem 1.4 and Lemma 6.1] there exists a definable $\mathcal{C}^{\infty}$ diffeomorphism $h_{0}: M \cap B_{1}(0) \rightarrow f\left(M \cap B_{1}(0)\right)$ such that $\left\|h_{0}-f\right\|_{m}<\varepsilon_{0}$ on $M \cap B_{1}(0)$.

Define $g_{0}: M \rightarrow N$ by

$$
g_{0}(x):= \begin{cases}h_{0}(x), & \text { if } x \in M \cap B_{1}(0), \\ f(x), & \text { otherwise. }\end{cases}
$$


Then the function $g_{0}$ is an $\mathcal{S}_{\text {sld }}^{m}$ diffeomorphism which evidently satisfies the properties (a)-(d) for $i=0$.

Suppose that $g_{i-1}$ is constructed. By [14, Theorem 1.4], there exits a definable $\mathcal{C}^{\infty}$ diffeomorphism $h_{i}: M \cap B(i-1, i+1) \rightarrow f(M \cap B(i-1, i+1))$ such that $\left\|h_{i}-g_{i-1}\right\|_{m}<\varepsilon_{i}$ on $M \cap B(i-1, i+1)$. Since $g_{i-1}$ is $\mathcal{C}^{\infty}$-smooth in $M \cap B_{i-1 / 4}(0)$, we may assume by [14, Lemma 6.1] that $h_{i}=g_{i-1}$ on $M \cap B(i-3 / 4, i-1 / 2)$. Define $g_{i}: M \rightarrow N$ by

$$
g_{i}(x):= \begin{cases}h_{i}(x), & \text { if } x \in M \cap B(i-1, i+1), \\ g_{i-1}(x), & \text { otherwise. }\end{cases}
$$

Then $g_{i}$ is an $\mathcal{S}_{\text {sld }}^{m}$ diffeomorphism from $M$ to $N$ which is $\mathcal{C}^{\infty}$-smooth in $M \cap B_{i+1}(0)$, and satisfies $\left\|g_{i}-g_{i-1}\right\|_{m}<\varepsilon_{i}$ on $M \cap B(i-1, i+1)$. Evidently $g_{i}=g_{i-1}$ outside of $M \cap B(i-3 / 4, i+1)$. Hence, item (a) is also satisfied. The limit $g:=\lim _{i \rightarrow \infty} g_{i}$ is the function we look for. Indeed, because of item (b) the function $g$ is an $\mathcal{S}_{\text {sld }}^{\infty}$ diffeomorphism, and item (a) implies that $g$ is close to $f$.

We say that two $\mathcal{S}^{m}$ manifolds $M$ and $N$ are $\mathcal{S}_{\text {sld }}^{m}$-diffeomorphic, if there is a $\mathcal{C}^{m}$ diffeomorphism $f: M \rightarrow N$ for which at least one of the functions $f$ or $f^{-1}$ is an $\mathcal{S}_{\text {sld }}$ function.

We note an immediate consequence of Theorem 5.5.

Corollary 5.6. Let $M$ and $N$ be $\mathcal{S}^{\infty}$ manifolds. Then $M$ and $N$ are $\mathcal{S}_{\text {sld }}^{\infty}$-diffeomorphic if and only if they are $\mathcal{S}_{\text {sld }}^{1}$-diffeomorphic.

Although we have stated and proved the previous theorem for $\mathcal{C}^{\infty}$-smooth approximation, the following statement is true.

REMARK 5.7. Let $\mathcal{M}$ be an arbitrary o-minimal expansion of the reals. Let $1 \leq m<\ell<\infty$. Let $M \subset \mathbb{R}^{n}$ and $N \subset R^{k}$ be $\mathcal{S}^{\ell}$ manifolds. Then every $\mathcal{S}_{\text {sld }}^{m}$ diffeomorphism $f: M \rightarrow N$ can be closely approximated by an $\mathcal{S}_{\text {sld }}^{\ell}$ diffeomorphism $g: M \rightarrow N$.

5.3. Diffeomorphisms and closed manifolds. We do not yet know any relation between $\mathcal{C}^{m}$ diffeomorphisms and $\mathcal{S}^{m}$ diffeomorphisms. In general, a $\mathcal{C}^{1}$ diffeomorphism between $\mathcal{S}^{1}$ manifolds does not imply that the manifolds are $\mathcal{S}^{1}$ diffeomorphic. Here, we will prove the density of $\mathcal{S}_{u}^{\infty}(M, N)$ in $\mathcal{C}^{1}(M, N)$ with respect to the Whitney topology if $M$ is a closed $\mathcal{S}$ manifold. This is based on the following observation.

Remark 5.8. Suppose $M$ is a closed $\mathcal{S}^{\infty}$ manifold. Then every continuous function $f: M \rightarrow \mathbb{R}$ is uniform. If $N$ is a further closed $\mathcal{S}^{\infty}$ manifold, then every diffeomorphism between them is bi-uniform. If both are compact this is trivial, and if both are non-compact, then, a diffeomorphism maps bounded sets on bounded sets, as compact sets are mapped on compact sets. The same holds for the inverse mapping. 
Next, we prove an approximation of arbitrary $\mathcal{C}^{m}$ functions from $\mathbb{R}^{n}$ to $\mathbb{R}$ by $\mathcal{C}^{\infty}$-smooth uniform strongly locally $\mathbb{R}_{\text {exp }}$-definable functions with respect to the strong Whitney topology. For analytic functions, this is a classical result of $\mathrm{H}$. Whitney. From the model-theoretic point of view, any analytic function $f: \mathbb{R}^{n} \rightarrow \mathbb{R}$ is a $\mathcal{C}^{\infty}$-smooth strongly locally $\mathbb{R}_{a n}$-definable function. The structure $\mathbb{R}_{\text {an }}$ expands $\overline{\mathbb{R}}$ by infinitely many functions, while the real exponential field is obtained by expanding the real field only by the graph of the exponential function.

We shall prove the following theorem.

TheOREm 5.9. Let $\mathcal{M}=\mathbb{R}_{\exp }$. Let $f: \mathbb{R}^{n} \rightarrow \mathbb{R}$ be a $\mathcal{C}^{m}$ function, and let $\varepsilon: \mathbb{R}^{n} \rightarrow(0, \infty)$ be a continuous function. Then there exists an $\mathcal{S}_{u}^{\infty}$ function $g: \mathbb{R}^{n} \rightarrow \mathbb{R}$ such that $\|f-g\|_{m}<\varepsilon$ on $\mathbb{R}^{n}$.

Proof. Select a $\mathcal{C}^{\infty}$-smooth $\mathbb{R}_{\text {exp }}$-definable function $\rho: \mathbb{R}^{n} \rightarrow \mathbb{R}$ which is strictly positive in $(-3 / 4,3 / 4)^{n}$ and which vanishes outside of $(-3 / 4,3 / 4)^{n}$. For $\alpha \in \mathbb{Z}^{n}$, let $Q_{\alpha} \subset \mathbb{R}^{n}$ denote the closed cube with side length $3 / 2$ and center $\alpha$. Then each $Q_{\alpha}$ intersects with $3^{n}-1$ other cubes $Q_{\beta}$.

Define for $\alpha \in Z^{n}$ the functions $\phi_{\alpha}: \mathbb{R}^{n} \rightarrow \mathbb{R}$ by

$$
\phi_{\alpha}(x):=\frac{\rho(x-\alpha)}{\sum_{\beta \in \mathbb{Z}^{n}} \rho(x-\beta)} .
$$

Then the functions $\phi_{\alpha}$ form a partition of unity. Moreover, each $\phi_{\alpha}$ is $\mathbb{R}_{\exp ^{-}}$ definable and the support of $\phi_{\alpha}$ is $Q_{\alpha}$. Furthermore, there is an $M>0$ such that $\left\|\phi_{\alpha}\right\|_{m}<M$ on $\mathbb{R}^{n}$ for any $\alpha \in \mathbb{Z}^{n}$. For every $\alpha \in \mathbb{Z}^{n}$, we choose by the Weierstrass approximation theorem a polynomial $p_{\alpha}$ such that

on $[-3,3]^{n}+\alpha$. Define $g: \mathbb{R}^{n} \rightarrow \mathbb{R}$ by

$$
\left\|f \phi_{\alpha}-\phi_{\alpha} p_{\alpha}\right\|_{m}<\frac{\inf \left\{\varepsilon(x) ; x \in[-3,3]^{n}+\alpha\right\}}{n^{m} M 3^{n}}
$$

$$
g(x):=\sum_{\alpha \in \mathbb{Z}^{n}} \phi_{\alpha} p_{\alpha} .
$$

Then $g$ is an $\mathcal{S}_{u}^{\infty}$ function. For $\alpha \in \mathbb{Z}^{n}$ let $S(\alpha)=\{-1,0,1\}^{n}+\alpha$. Moreover, for every $\alpha \in \mathbb{Z}^{n}$, we have on $Q_{\alpha}$ :

$$
\begin{aligned}
\|f-g\|_{m} & =\left\|f-\sum_{\beta \in S(\alpha)} \phi_{\beta} p_{\beta}\right\|_{m} \\
& =\left\|\sum_{\beta \in S(\alpha)}\left(f-p_{\beta}\right) \phi_{\beta}\right\|_{m} \\
& \leq \sum_{\beta \in S(\alpha)}\left\|\left(f-p_{\beta}\right) \phi_{\beta}\right\|_{m} \\
& \leq \sum_{\beta \in S(\alpha)} n^{m}\left\|\phi_{\beta}\right\|_{m}\left\|\left(f-p_{\beta}\right)\right\|_{m}
\end{aligned}
$$




$$
\begin{aligned}
& <\sum_{\beta \in S(\alpha)} n^{m} M \frac{\inf \left\{\varepsilon(x) ; x \in[-3 / 2,3 / 2]^{n}+\alpha\right\}}{n^{m} M 3^{n}} \\
& =\inf \left\{\varepsilon(x) ; x \in[-3 / 2,3 / 2]^{n}+\alpha\right\} .
\end{aligned}
$$

Hence, $\|f-g\|_{m}<\varepsilon$ on $Q_{\alpha}$ for every $\alpha \in \mathbb{Z}^{n}$.

We note the following immediate corollary of Theorem 5.9.

Corollary 5.10. The set of $\mathcal{S}_{u}^{\infty}$ functions from $\mathbb{R}^{n}$ to $\mathbb{R}$ is dense in $\mathcal{C}^{m}\left(\mathbb{R}^{n}, \mathbb{R}\right)$ with respect to the $\mathcal{C}^{m}$-topology.

This density also holds for closed manifolds.

Proposition 5.11. Let $M \subset \mathbb{R}^{n}$ be a closed $\mathcal{S}^{\infty}$ manifold. Then $\mathcal{S}_{u}^{\infty}(M, \mathbb{R})$ is dense in $\mathcal{C}^{m}(M, \mathbb{R})$ with respect to the $\mathcal{C}^{m}$-topology.

Proof. Let $f \in \mathcal{C}^{m}(M, \mathbb{R})$. By Whitney's extension theorem, the function $f$ is the restriction of a $\mathcal{C}^{m}$ function $F: \mathbb{R}^{n} \rightarrow \mathbb{R}$ to $M$. Approximate this function by an $\mathcal{S}_{u}^{\infty}$ function $G: \mathbb{R}^{n} \rightarrow \mathbb{R}$. Then $g:=\left.G\right|_{M}$ is sufficiently close, if we choose $G$ sufficiently close to $F$.

This allow us to state a more general separation of sets.

Corollary 5.12. Let $M \subset \mathbb{R}^{n}$ be a closed $\mathcal{S}^{\infty}$ manifold, and let $A$ and $B$ be two closed disjoint subsets. Then there is an $\mathcal{S}_{u}^{\infty}$ function $\phi$ with

$$
A \subset\{\phi=1\} \text { and } B \subset\{\phi=0\} .
$$

Proof. Using classical methods, there is a $\mathcal{C}^{\infty}$ function $\Phi: M \rightarrow \mathbb{R}$ such that $A=\{\Phi=-1\}$ and $B=\{\Phi=2\}$. Choose an $\mathcal{S}_{u}^{\infty}$ function $\phi: M \rightarrow \mathbb{R}$ for which $\|\phi-\Phi\|_{0}<1$ on $M$ holds true. Then $\phi$ satisfies equation (3).

The following theorem provides us with a criterion to decide whether two closed $\mathcal{S}^{\infty}$ manifolds are bi-uniformly $\mathcal{S}_{u}^{\infty}$-diffeomorphic.

THEOREM 5.13. Let $M$ and $N$ be closed $\mathcal{S}^{\infty}$ manifolds. Then $M$ and $N$ are $\mathcal{S}_{u}^{\infty}$-diffeomorphic if and only if they are $\mathcal{C}^{1}$-diffeomorphic.

Proof. Let $f: M \rightarrow N$ be a $\mathcal{C}^{1}$ diffeomorphism. If $M$ is compact, then $N$ is also compact, and the statement of the theorem is a consequence of the Weierstrass approximation theorem. So we may assume that $M$ is not compact. Then $f$ and $f^{-1}$ are uniform. By Theorem 5.9, the $\mathcal{S}_{u}^{\infty}$ functions from $M$ to $N$ are dense in $\mathcal{C}^{1}(M, N)$ with respect to the $\mathcal{C}^{1}$-topology. Thus, a sufficiently close $\mathcal{S}_{u}^{\infty}$ approximation $g$ of $f$ is $\mathcal{S}_{u}^{\infty}$ diffeomorphism from $M$ to $N$. 


\section{Diffeomorphism classes}

In analogy to diffeomorphism classes in classical differential geometry, we define diffeomorphism classes for $\mathcal{S}$ manifolds. We commence with uniform diffeomorphism classes.

Definition 6.1. Let $1 \leq m$ be an integer, or let $m=\infty$. Two $\mathcal{S}^{m}$ manifolds $M$ and $N$ are called $\mathcal{S}_{u}^{m}$-equivalent if there exists a bi-uniform $\mathcal{S}^{m}$ diffeomorphism between $M$ and $N$. An $\mathcal{S}_{u}^{m}$ diffeomorphism class is the collection of all $\mathcal{S}^{m}$ manifolds which are bi-uniformly $\mathcal{S}_{u}^{m}$-diffeomorphic to one given $\mathcal{S}^{m}$ manifold.

Since compositions of $\mathcal{S}_{u}$ functions are $\mathcal{S}_{u}$ functions, the manifolds in an $\mathcal{S}_{u}^{m}$ diffeomorphism class are $\mathcal{S}_{u}^{m}$ equivalent. In the following, we investigate some properties of $\mathcal{S}_{u}^{m}$ diffeomorphism classes. First, we prove the following fundamental theorem.

TheOREM 6.2. Let $M$ be an $\mathcal{S}^{m}$ manifold. Then there is an $\mathcal{S}^{\infty}$ manifold $N$ and a bi-uniform $\mathcal{S}^{m}$ diffeomorphism $\phi: M \rightarrow N$ which may be chosen $\mathcal{S}_{u}^{m}$-close to identity.

Proof. Let $\varepsilon: M \rightarrow(0, \infty)$ be a continuous $\mathcal{S}$ function. For any $r>0$, let $B_{r}$ denote the open ball with radius $r$ and center 0 , and let $B_{0}=\varnothing$. We construct a sequence of $\mathcal{S}^{m}$ manifolds $M_{0}, M_{1}, \ldots$ and $\mathcal{S}_{u}^{m}$ diffeomorphism $\phi_{0}: M_{i} \rightarrow M_{i+1}$ such that $f$ or all $i$ :

(a) $M_{i} \cap B_{i}$ is a $\mathcal{C}^{\infty}$-smooth manifold.

(b) $\phi_{i}$ is identity on $M_{i} \cap B_{i-1}$ and outside of $M_{i} \cap B_{i+1}$.

(c) $\left\|\phi_{i}-\mathrm{id}\right\|_{m}<\varepsilon / 3$ on $M_{i}$.

We may assume that $0 \notin M$. Set $M_{0}:=M$, and $\phi_{-1}=$ id. Suppose now that for $j \leq i$, the manifolds $M_{j}$ and for $j<i$ the diffeomorphisms are constructed. Consider $N_{i}:=M_{i} \cap B_{i+1}$. This is a definable $\mathcal{C}^{m}$ manifold. Let $\delta: N_{i} \rightarrow(0,1)$ be defined by

$$
\delta(x):=\min \left(\frac{1}{3} \varepsilon(x), \operatorname{dist}\left(x, \partial N_{i}\right), \operatorname{dist}\left(x, B_{i+1}\right)\right) .
$$

By [14, Theorem 1.5] applied to $N_{i}$ and $N_{i} \backslash B_{i-1 / 2}$ in place of $M$ and $V$, we obtain a $\mathcal{C}^{\infty}$-smooth definable manifold $P_{i}$ and a definable $\mathcal{C}^{m}$ diffeomorphism $\psi_{i}: N_{i} \rightarrow P_{i}$ such that $\left\|\psi_{i}-\mathrm{id}\right\|_{m}<\delta$ on $N_{i}$, and $\psi_{i}$ restricted to $N_{i} \cap B_{i-1 / 2}$ is identity. Hence, we may define the $\mathcal{S}^{m}$ manifold $M_{i+1}$ as the union of $P_{i}$ and $M_{i} \backslash B_{i+1}$ and $\phi_{i}: M_{i} \rightarrow M_{i+1}$ by

$$
\phi_{i}(x):= \begin{cases}x, & \text { if } x \in M_{i} \backslash B_{i+1}, \\ \psi_{i}(x), & \text { if } x \in M_{i} \cap B_{i+1} .\end{cases}
$$


So $\left\|\phi_{i}-\mathrm{id}\right\|_{m}<\varepsilon / 3$ on $M_{i}$ and $\phi_{i}$ restricted to $M_{i} \cap B_{i+1}$ and $M_{i} \backslash B_{i+1}$ is identity. Hence, by construction,

$$
\left\|\phi_{i} \circ \phi_{i-1} \circ \cdots \circ \phi_{0}-\mathrm{id}\right\|_{m}<\varepsilon
$$

on $M$. The limit $\phi:=\lim _{i \rightarrow \infty} \phi_{i} \circ \phi_{i-1} \circ \cdots \circ \phi_{0}$ is a bi-uniform $\mathcal{S}^{m}$ diffeomorphism and $\|\phi-\mathrm{id}\|_{m}<\varepsilon$ on $M$.

As a consequence, we obtain the following statements about $\mathcal{S}_{u}^{m}$ diffeomorphism classes.

Corollary 6.3. Let $T$ be an $\mathcal{S}_{u}^{m}$ diffeomorphism class. Then $T$ contains an $\mathcal{S}^{\infty}$ manifold.

COROLLARY 6.4. Let $T$ be an $\mathcal{S}_{u}^{m}$ diffeomorphism class of $\mathcal{S}^{m}$ submanifolds of $\mathbb{R}^{n}$. Then $T$ contains an $\mathcal{S}^{\infty}$ manifold.

It is evident that the cardinal number of an $\mathcal{S}_{u}^{m}$ diffeomorphism class can have at least the power of continuum. But also the set of all diffeomorphism classes has at least the power of continuum, as we shall prove in the following proposition.

Proposition 6.5. Let $1 \leq m$ be an integer, or let $m=\infty$. The set of $\mathcal{S}_{u}^{m}$ diffeomorphism classes has at least the power of continuum.

Proof. We construct a set of pairwise non- $\mathcal{C}^{1}$-diffeomorphic $\mathcal{S}^{\infty}$ manifolds which has the power of continuum. For $n \in \mathbb{N}$, let $Q_{n} \subset \mathbb{R}^{2}$ denote the open square with vertices $(n-1,0),(n-1,1),(n, 0)$ and $(n, 1)$. For a sequence $s=\left(s_{n}\right)_{n \in \mathbb{N}} \in\{0,1\}^{\mathbb{N}}$, we let $Q_{n}^{s}$ be the square $Q_{n}$ from which we take off $2 n+s_{n}$ points. Let $s, t \in\{0,1\}^{\mathbb{N}}$. Then $Q_{n}^{s}$ and $Q_{m}^{t}$ cannot be diffeomorphic if $n \neq m$, and, if $s_{n} \neq t_{n}$ for some $n$, then $Q_{n}^{s}$ and $Q_{n}^{t}$ cannot be diffeomorphic.

Having in mind that the sets $Q_{n}^{s}$ and $Q_{m}^{s}$ are disjoint for $n \neq m$, we define the $\mathcal{S}^{\infty}$ manifold $M_{s}$ by $M_{s}:=\bigcup_{n \in \mathbb{N}} Q_{n}^{s}$. For $s, t \in\{0,1\}^{\mathbb{N}}$ with $s \neq t$, the manifolds $M_{s}$ and $M_{t}$ are not diffeomorphic. So the map assigning to each $s$ the diffeomorphism class containing $M_{s}$ is injective. As $\{0,1\}^{\mathbb{N}}$ has the power of continuum, the set of $\mathcal{S}_{u}^{\infty}$ diffeomorphism classes has at least the power of continuum.

The proof of the previous proposition has shown more. Therefore, we the following version of diffeomorphism classes.

Definition 6.6. Let $m$ be a positive integer or $m=\infty$.

(a) Two $\mathcal{S}^{m}$ manifolds are called $\mathcal{C}^{m}$-equivalent if they are $\mathcal{C}^{m}$-diffeomorphic.

(b) A $\mathcal{C}_{S}^{m}$ diffeomorphism class is the collection of all $\mathcal{S}^{m}$ manifolds which are $\mathcal{C}^{m}$-diffeomorphic to one given $\mathcal{S}^{m}$ manifold.

In the proof of Proposition 6.5, we have also proved the following corollary.

Corollary 6.7. Let $1 \leq m$ be an integer, or let $m=\infty$. The set of $\mathcal{C}_{S}^{m}$ diffeomorphism classes has at least the power of continuum. 
For $m \geq 1$, every $\mathcal{S}_{u}^{m}$ diffeomorphism class is contained in some $\mathcal{C}_{S}^{m}$ diffeomorphism class. The amount of $\mathcal{S}_{u}^{m}$ diffeomorphism classes contained in some $\mathcal{C}_{S}^{m}$ diffeomorphism class can have the power of continuum.

Proposition 6.8. There is a $\mathcal{C}_{S}^{m}$ diffeomorphism class $D$ for which the cardinal number of the set of $\mathcal{S}_{u}^{m}$ diffeomorphism classes contained in $D$ has at least the power of continuum

Proof. For $n \in \mathbb{N}$, let $Q_{n} \subset \mathbb{R}^{2}$ denote the open cube with the vertices $(n, 0)$, $(n, 1),(n+1,0)$ and $(n+1,1)$ minus $n$ points. Set $P_{n}(0)=Q_{n}$ and $P_{n}(1)=$ $\tau\left(Q_{n}\right)$ where $\tau: \mathbb{R} \times(-1,1) \rightarrow \mathbb{R}^{2}$ is the function $\tau(x, y)=\left(x, y / \sqrt{1-y^{2}}\right)$. Then $P_{n}(1)$ and $P_{n}(0)$ are diffeomorphic, but $P_{n}(i)$ and $P_{m}(j)$ are not diffeomorphic for any choice of $i$ and $j$ and $m \neq n$. Therefore, if $\left(s_{n}\right)_{n \in \mathbb{N}}$, $\left(t_{n}\right)_{n \in \mathbb{N}} \in\{0,1\}^{\mathbb{N}}$ are different sequences, the $\mathcal{S}^{\infty}$ manifolds $\bigcup_{n \in \mathbb{N}} P_{n}\left(s_{n}\right)$ and $\bigcup_{n \in \mathbb{N}} P_{n}\left(t_{n}\right)$ are not $\mathcal{S}_{u}^{\infty}$-diffeomorphic. However, they are $\mathcal{C}^{m}$-diffeomorphic.

In the final Section 7, we will be able to describe $\mathcal{C}_{S}^{m}$ diffeomorphism classes using only $\mathcal{S}^{m}$ diffeomorphisms.

\section{Sums and compositions of $\mathcal{S}$ functions}

It is somewhat dissatisfying that a bounded open interval and the real line are not in the same $\mathcal{S}_{u}^{1}$ diffeomorphism class although they are definably diffeomorphic. In this section, we study sums and compositions of $\mathcal{S}$ functions. Notice that, in general, neither a sum nor a composition of $\mathcal{S}$ functions is an $\mathcal{S}$ function.

7.1. Locally definable functions. To obtain a better understanding of this phenomenon, we use a weaker concept of locally definable function.

Definition 7.1.

(a) A set $X \subset \mathbb{R}^{n}$ is called locally definable, if for every point $x \in X$ there exists an open ball $B$ containing $x$ such that $B \cap X$ is definable.

(b) A function $f$ between locally definable sets $X$ and $Y$ is called locally definable, if for every $x \in X$ there exists a ball $B$ containing $x$ such that $f$ restricted to $B \cap X$ is definable.

We denote the locally definable $\mathcal{C}^{m}$ functions from $U$ to $W$ by $\mathcal{S}_{\text {loc }}^{m}(U, W)$.

It is evident that a closed locally definable set is an $\mathcal{S}$ set and, if $M$ is a closed $\mathcal{S}$ set and $f: M \rightarrow \mathbb{R}^{n}$ is a continuous locally definable function, then $f$ is actually an $\mathcal{S}_{u}$ function. The locally definable functions are closed under composition of which we assure ourselves next.

Proposition 7.2. Let $M, N$ and $P$ be locally definable sets. Let $f: M \rightarrow$ $N$ and $g: N \rightarrow P$ be locally definable continuous functions. Then the composition $g \circ f$ is locally definable. 
Proof. Let $x \in M$, and $y=f(x)$. then there is an $\varepsilon>0$ such that $g$ restricted to $B_{\varepsilon}(y) \cap N$ is a definable function. The continuity of $f$ implies that there is a $\delta>0$ such that $f\left(B_{\delta}(x)\right) \subset B_{\varepsilon}(y)$. By a further shrinking of $\delta$, we may assume that $f$ restricted to $B_{\delta}(x)$ is definable. Then $g \circ f$ restricted to $B_{\delta}(x)$ is definable.

Since addition and multiplication are locally definable functions, the set $\mathcal{S}_{\text {loc }}^{m}(M, \mathbb{R})$ forms a ring with addition and multiplication of functions.

7.2. Sums. The locally definable functions are not arbitrarily chosen. They can be constructed from the $\mathcal{S}^{m}$ functions in several ways. We show their algebraic construction starting with $\mathcal{S}^{m}(M, \mathbb{R})$.

Proposition 7.3. Let $m \in \mathbb{N} \cup\{\infty\}$, and let $M \subset \mathbb{R}^{n}$ be an $\mathcal{S}^{m}$ manifold. The group $\left(\mathcal{S}_{\text {loc }}^{m}(M, \mathbb{R}),+\right)$ is the smallest group containing $\left(\mathcal{S}^{m}(M, \mathbb{R}),+\right)$.

Proof. Let $f \in \mathcal{S}_{\text {loc }}^{m}(M, \mathbb{R})$. Then $(f-1)^{2}$ and $(f+1)^{2}$ belong to $\mathcal{S}_{\text {loc }}^{m}(M, \mathbb{R})$. By the identity (1) we see that every $\mathcal{S}_{\text {loc }}^{m}$ function is a difference of nonnegative $\mathcal{S}_{\text {loc }}^{m}$ functions. Hence, may assume that $f \geq 0$ on $M$. Let $U=\mathbb{R}^{n} \backslash(\partial M)$. By Corollary 5.2, there is an $\mathcal{S}_{u}^{m}$ function $g: U \rightarrow(0, \infty)$ which satisfies

$$
\|g-\operatorname{dist}(-, \partial M)\|_{0}<\frac{1}{2} \operatorname{dist}(-, \partial M)
$$

on $M$. Then $1 / g$ is an $\mathcal{S}_{\text {sld }}^{m}$ function. Let $h:=f+1 / g$. Then $h$ is an $\mathcal{S}_{\text {loc }}^{m}$ function whose graph is closed. So $h$ is an $\mathcal{S}^{m}$ function, and

$$
f=h-1 / g \text {. }
$$

The proof of the previous proposition also implies the following notable corollary.

Corollary 7.4. Every $\mathcal{S}_{\text {loc }}^{m}$ function is the sum of two $\mathcal{S}^{m}$ functions.

Proof. By equation (1), every $\mathcal{S}_{\text {loc }}^{m}$ function $f$ is the difference of two nonnegative $\mathcal{S}_{\text {loc }}^{m}$ functions $f_{1}$ and $f_{2}$. Then, by equation (4), we have $f_{i}=$ $h_{i}-1 / g_{i}$ for $i=1,2$, where $h_{1}$ and $h_{2}$ are $\mathcal{S}^{m}$ functions. Note that the choice of the function $g$ in the proof of Proposition 7.3 depends only on the domain $U$, so we may assume that $g_{1}=g_{2}$, so $f=h_{1}+\left(-h_{2}\right)$.

For subanalytic functions, we may note that any analytic function with subanalytic domain is the sum of two analytic subanalytic function. This has been shown in [16]. A finite sum of locally definable functions is again a locally definable function. Hence, a finite sum of $\mathcal{S}$ functions is a locally definable function. The previous corollary implies the following one.

Corollary 7.5. Let $M \subset \mathbb{R}^{n}$ be an $\mathcal{S}^{m}$ manifold, and let $f_{1}, \ldots, f_{r}: M \rightarrow$ $\mathbb{R}^{k}$ be $\mathcal{S}^{m}$ functions. Then there are $g_{1}, g_{2} \in \mathcal{S}^{m}\left(M, \mathbb{R}^{k}\right)$ such that $f_{1}+\cdots+$ $f_{r}=g_{1}+g_{2}$. 
7.3. Compositions. In the subanalytic setting, every analytic function with subanalytic domain is the composition of two analytic subanalytic functions, see [15]. In the present situation, we obtain a stronger version for the decomposition of locally definable $\mathcal{C}^{\infty}$-smooth functions into compositions of $\mathcal{C}^{\infty}$-smooth $\mathcal{S}_{\text {sld }}$ functions. We shall prove the following theorem.

Theorem 7.6. Let $M$ and $N$ be $\mathcal{S}^{m}$ manifolds, and let $f: M \rightarrow N$ be a locally definable $\mathcal{C}^{m}$ function. Then there are:

(a) a closed $\mathcal{S}^{m}$ manifold $P$,

(b) an $\mathcal{S}_{\text {sld }}^{m}$ diffeomorphism $\phi: M \rightarrow P$ whose inverse $\phi^{-1}$ is an $\mathcal{S}_{u}^{m}$ function, (c) an $\mathcal{S}_{u}^{m}$ function $\psi: P \rightarrow N$,

such that $f=\psi \circ \phi$.

Proof. Select by Corollary 5.2 an $\mathcal{S}_{u}^{m}$ function $h: M \rightarrow(0, \infty)$ such that $h(x) \rightarrow 0$ as $x \rightarrow \partial M$ or $\|x\| \rightarrow \infty$. Define $P$ as $P:=\{(x, t) \in M \times \mathbb{R}$; $h(x) t=1\}$. Then $P$ is a closed $\mathcal{S}^{m}$ manifold, and the map $\phi: M \rightarrow P$,

$$
\phi(x):=\left(x, \frac{1}{h(x)}\right)
$$

is an $\mathcal{S}_{\text {sld }}^{m}$ diffeomorphism whose inverse is the projection on $M$. Therefore $\phi$ is an $\mathcal{S}_{u}^{m}$ function. Let $\psi:=f \circ \pi$. Since the composition of locally definable maps is again locally definable, and $P$ is a closed set, the function $\psi$ is an $\mathcal{S}_{u}^{m}$ function. Evidently we have $\psi \circ \phi=f \circ \pi \circ \phi=f$.

The previous theorem allows us to get a better understanding of compositions of $\mathcal{S}^{m}$ functions.

Corollary 7.7. Let $M_{1}, \ldots, M_{r}$ be $\mathcal{S}^{m}$ manifolds and let $f_{i}: M_{i} \rightarrow M_{i+1}$ be an $\mathcal{S}^{m}$ function for $i=1, \ldots, r-1$. Then there are a closed $\mathcal{S}^{m}$ manifold $N$, an $\mathcal{S}_{\text {sld }}^{m}$ diffeomorphism $\phi: M_{1} \rightarrow N$ and an $\mathcal{S}_{u}^{m}$ function $\psi: N \rightarrow M_{r}$ such that

$$
f_{r} \circ \cdots \circ f_{1}=\psi \circ \psi \text {. }
$$

Moreover, the class of $\mathcal{S}_{\text {loc }}$ functions is very rich. We actually have the following corollary.

Corollary 7.8. Let $M$ and $N$ be $\mathcal{S}^{m}$ manifolds. Then $M$ and $N$ are locally definably $\mathcal{C}^{m}$-diffeomorphic if and only if they are $\mathcal{C}^{1}$-diffeomorphic.

Proof. By the proof of Theorem 7.6, both manifolds $M$ and $N$ are $\mathcal{S}^{m}$ diffeomorphic to closed $\mathcal{S}^{m}$ manifolds $P$ and $Q$, respectively. By Theorem 6.2, both $P$ and $Q$ are $\mathcal{S}^{m}$-diffeomorphic to closed $\mathcal{C}^{\infty}$-smooth $\mathcal{S}$ manifolds $R$ and $S$, respectively. Since $R$ and $S$ are $\mathcal{C}^{1}$-diffeomorphic, they are $\mathcal{S}_{u}^{\infty}$ diffeomorphic by Theorem 5.13. Hence, since compositions of locally definable functions are locally definable functions, the manifolds $M$ and $N$ are locally definably $\mathcal{C}^{m}$-diffeomorphic. 
This leads to the following corollary.

Corollary 7.9. Let $M$ and $N$ be $\mathcal{S}^{m}$ manifolds which are $\mathcal{C}^{1}$-diffeomorphic. Then there is a closed $\mathcal{S}^{m}$ manifold $P$, an $\mathcal{S}_{\text {sld }}^{m}$ diffeomorphism $\phi: M \rightarrow$ $P$ and a uniform $\mathcal{S}^{m}$ diffeomorphism $\psi: P \rightarrow N$.

Finally we obtain an alternative description of $\mathcal{C}_{S}^{m}$ diffeomorphism class by means of $\mathcal{S}_{\text {sld }}$ diffeomorphisms.

Corollary 7.10. For every $\mathcal{C}_{S}^{m}$ diffeomorphism class $C$, there exists a closed $\mathcal{C}^{\infty}$-smooth $\mathcal{S}$ manifold $P \in C$ such that every manifold $M \in C$ is $\mathcal{S}_{\text {sld }^{-}}$ diffeomorphic to $P$.

7.4. Sheaves. The concept of sheaves needs to be adopted to the $\mathcal{S}$ setting.

Definition 7.11. An $\mathcal{S}$-sheaf $\mathcal{F}$ on an $\mathcal{S}$ set $U$ consists of the following data.

(a) For every open $\mathcal{S}$ set $X \subset U$ there is a sub- $\mathbb{R}$-algebra $\mathcal{F}(X)$ of continuous $\mathcal{S}_{\text {sld }}$ functions from $X$ to $\mathbb{R}$ given.

(b) For every inclusion $X \subset Y \subset U$ of open $\mathcal{S}$ sets, the inclusion map $\iota: X \rightarrow$ $Y$ induces an $\mathbb{R}$-algebra homomorphism $h: \mathcal{F}(Y) \rightarrow \mathcal{F}(X)$ by $h(f)=f \circ \iota$.

(c) Let $\left\{U_{i} ; i \in \mathbb{N}\right\}$ be an open $\mathcal{S}$-cover of $U$. Suppose that for each $i \in \mathbb{N}$ there is an $f_{i} \in \mathcal{F}\left(U_{i}\right)$ such that for $i, j \in \mathbb{N}$ the functions $f_{i}$ and $f_{j}$ coincide on $U_{i} \cap U_{j}$. Then there is an $f \in \mathcal{F}(U)$ such that $f=f_{i}$ on $U_{i}$.

A morphism of $\mathcal{S}$-sheaves is defined as follows.

Definition 7.12. A morphism of $\mathcal{S}$-sheafs from $\mathcal{F}$ (on $U$ ) to $\mathcal{G}$ on $(V)$ consists of a continuous $\mathcal{S}$ function $f: V \rightarrow U$ for which inverse images of $\mathcal{S}$ sets are $\mathcal{S}$ sets such that for every $\mathcal{S}$ set $U^{\prime} \subset U$ there is an $\mathbb{R}$-algebra homomorphism $\theta^{\prime}: \mathcal{F}\left(U^{\prime}\right) \rightarrow \mathcal{G}\left(f^{-1}\left(U^{\prime}\right)\right)$ given by

$$
\theta^{\prime}(h)=h \circ f
$$

restricted to $f^{-1}\left(U^{\prime}\right)$. The collection of $\mathbb{R}$-algebra homomorphisms is denoted by $\theta$. In short we say that $(f, \theta): \mathcal{F} \rightarrow \mathcal{G}$ is an $\mathcal{S}$-sheaf morphism.

REMARK 7.13. If we replace $\mathbb{R}$-algebra by $\mathbb{R}$-vector space in the previous definitions we obtain $\mathcal{S}$-sheaves of vector spaces and its corresponding morphisms.

To claim that the $\mathcal{S}$ function $f$ of the previous definition imply that preimages of $\mathcal{S}$ sets are $\mathcal{S}$ set is not superfluous. Not even the class of strongly locally definable functions satisfies this property. We shall need the following proposition.

Proposition 7.14. Let $\mathcal{M}$ be an o-minimal expansion of $\mathbb{R}_{\exp }$. Let $f: V \rightarrow$ $U$ be a continuous $\mathcal{S}$ function which is not uniform, where $U$ is an $\mathcal{S}^{\infty}$ manifold. Then there is a bounded $\mathcal{S}^{\infty}$ function $g: U \rightarrow \mathbb{R}$ such that $g \circ f$ is not an $\mathcal{S}$ function. 
Proof. Consider the definable function $h: \mathbb{R} \rightarrow[0,1]$, defined by

$$
h(t):=e^{4} e^{-1 / t} e^{1 /(t-1)} \chi_{[0,1]}(t) .
$$

Then $g: \mathbb{R} \rightarrow[0,1]$, defined by

$$
\tilde{g}(t):=\sum_{z \in \mathbb{Z}} h(t-z)
$$

is a bounded, periodic $\mathcal{S}^{\infty}$ function. In particular, we have $\tilde{g}(z)=0$ and $\tilde{g}(1 / 2+z)=1$ for $z \in \mathbb{Z}$. Define $g: U \rightarrow[0,1]$ by $g(x):=\tilde{g}\left(\|x\|^{2}\right)$. Since $f$ is not uniform, there exists a bounded $\mathcal{S}$ set $V^{\prime}$ of $V$, such that $f\left(V^{\prime}\right)$ is not bounded. Since $V^{\prime}$ is definable, we may assume that $V^{\prime}$ is even connected. Therefore, $f\left(V^{\prime}\right)$ is a connected unbounded set. We assume that $g \circ f$ is an $\mathcal{S}$ function. Then $g \circ f$ is a bounded $\mathcal{S}$ function so that its restriction to $V^{\prime}$ is actually definable. Hence $(g \circ f)^{-1}(1)$ is a definable set, and therefore it has only finitely many connected components. On the other hand, the set $\left.g\right|_{f\left(V^{\prime}\right)} ^{-1}(1)$ has infinitely many connected components. Thus $g \circ f$ is no $\mathcal{S}$ function.

We apply this proposition to morphisms of $\mathcal{S}$-sheaves in the proof of the following corollary.

Corollary 7.15. Let $\mathcal{F}$ and $\mathcal{G}$ be $\mathcal{S}$-sheafs of functions on $U \subset \mathbb{R}^{n}$ and $V$, respectively. Let $(f, \theta): \mathcal{F} \rightarrow \mathcal{G}$ be a morphism of $\mathcal{S}$ sheafs such that $\mathcal{F}(U)$ contains all the restrictions of bounded $\mathcal{S}^{\infty}$ functions on $\mathbb{R}^{n}$ to $U$. Then $f: V \rightarrow U$ is a uniform continuous $\mathcal{S}$ function.

Proof. Suppose that $f$ is not uniform. Since $\mathcal{F}(U)$ contains all bounded $\mathcal{S}^{\infty}$ functions, there is a bounded $\mathcal{S}^{\infty}$ function $g \in \mathcal{F}(U)$, such that $g \circ f$ is not an $\mathcal{S}$ function by Proposition 7.14. But then, for $U^{\prime}=U$, the function $\theta^{\prime}(g)=g \circ f \notin \mathcal{G}(V)$. A contradiction.

As a consequence of Proposition 3.2, we note the following corollary.

Corollary 7.16. Let $M$ and $N$ be two $\mathcal{C}^{\infty}$-smooth $\mathcal{S}$ manifolds, and let $f: M \rightarrow N$ be a $\mathcal{C}^{\infty}$-smooth uniform $\mathcal{S}$ function. Then

$$
g \mapsto g \circ f
$$

is an $\mathbb{R}$-algebra homomorphism from the $\mathbb{R}$-algebra of $\mathcal{C}^{\infty}$-smooth strongly locally definable functions on $N$ to the $\mathcal{C}^{\infty}$-smooth strongly locally definable functions on $M$. Its restriction to the $\mathcal{C}^{\infty}$-smooth uniform $\mathcal{S}^{\infty}$ functions is also an $\mathbb{R}$-algebra homomorphism.

We already know that bi-uniform $\mathcal{S}^{\infty}$-diffeomorphisms are closed under compositions. Thus, Proposition 3.2 implies the following corollary. 
Corollary 7.17. Let $M$ and $N$ be two $\mathcal{C}^{\infty}$-smooth $\mathcal{S}$ manifolds, and let $f: M \rightarrow N$ be a $\mathcal{C}^{\infty}$-smooth $\mathcal{S}$ function. Then

$$
g \mapsto g \circ f
$$

is an $\mathbb{R}$-algebra isomorphism from the $\mathbb{R}$-algebra of $\mathcal{C}^{\infty}$-smooth strongly locally definable functions on $N$ to the $\mathcal{C}^{\infty}$-smooth strongly locally definable functions on $M$ if and only if $f$ is a bi-uniform $\mathcal{S}^{\infty}$ diffeomorphism.

Proof. Corollary 7.16 implies that the bi-uniformity of $f$ is necessary, and Proposition 3.2 says that the bi-uniformity is sufficient.

We continue with the following consequence of Proposition 3.3.

Corollary 7.18. Let $\theta_{1}, \theta_{2}: \mathcal{S}_{\text {sld }}^{\infty}(U, \mathbb{R}) \rightarrow \mathcal{S}_{\text {sld }}^{\infty}(V, \mathbb{R})$ be two $\mathbb{R}$-algebra homomorphisms which coincide on $\mathcal{S}_{u}^{\infty}(U, \mathbb{R})$. Then $\theta_{1}=\theta_{2}$.

Proof. Both homomorphisms $\theta_{1}$ and $\theta_{2}$ map units on units, and the set $W$ from Proposition 3.3 consists only of units in $\mathcal{S}_{\text {sld }}^{\infty}(U, \mathbb{R})$. Since $\mathcal{S}_{\text {sld }}^{\infty}(U, \mathbb{R})$ is the localization of $\mathcal{S}_{u}^{\infty}(U, \mathbb{R})$ with respect to $W$, the statement of the corollary follows.

It is worth mentioning that an $\mathbb{R}$-algebra homomorphism form $\mathcal{S}_{\text {sld }}^{\infty}(U, \mathbb{R})$ to $\mathcal{S}_{\text {sld }}^{\infty}(V, \mathbb{R})$ always maps strictly positive functions on strictly positive functions, as these functions are squares and units. We do not know if every $\mathbb{R}$-algebra homomorphism between algebras of $\mathcal{S}_{u}^{\infty}$ functions maps strictly positive functions on strictly positive functions. If an $\mathbb{R}$-algebra homomorphism maps a strictly positive unit to a unit, this unit is strictly positive, since every strictly positive uniform $\mathcal{S}^{\infty}$ function is the square of a strictly positive uniform $\mathcal{S}^{\infty}$ function. However, the image of a strictly positive function is a square.

COROLlary 7.19. Let $\theta: \mathcal{S}_{u}^{\infty}(U, \mathbb{R}) \rightarrow \mathcal{S}_{u}^{\infty}(V, \mathbb{R})$ be an $\mathbb{R}$-algebra homomorphism, which maps strictly positive functions on strictly positive functions. Then $\theta$ lifts up to a unique $\mathbb{R}$-algebra homomorphism from $\mathcal{S}_{\text {sld }}^{\infty}(U, \mathbb{R}) \rightarrow$ $\mathcal{S}_{\text {sld }}^{\infty}(V, \mathbb{R})$.

Another observation is the following. An $\mathbb{R}$-algebra homomorphism $\Theta$ : $\mathcal{S}_{\text {sld }}^{\infty}(U, \mathbb{R}) \rightarrow \mathcal{S}_{\text {sld }}^{\infty}(V, \mathbb{R})$ maps bounded functions to bounded functions. If $f \in$ $\mathcal{S}_{\text {sld }}^{\infty}(U, \mathbb{R}) \rightarrow$ and there is a $c \in \mathbb{R}$ such that $|f|<c$, then $c-f>0$, hence $\Theta(c-f)>0$, and therefore $\Theta(f)<c$ and similarly $\Theta(f)>-c$. Unfortunately, we do not know if $\Theta$ maps uniform functions on uniform functions. However, on the level of $\mathcal{S}$-sheafs, we do have the following correspondence.

Proposition 7.20.

(a) Let $(\theta, f): \mathcal{S}_{u}^{\infty}(U, \mathbb{R}) \rightarrow \mathcal{S}_{u}^{\infty}(V, \mathbb{R})$ be a morphism of $\mathcal{S}$-sheafs. Then $(\theta, f)$ lifts up to a uniquely determined morphism of $\mathcal{S}$-sheaves $(\Theta, f): \mathcal{S}_{\text {sld }}^{\infty}(U$, $\mathbb{R}) \rightarrow \mathcal{S}_{\text {sld }}^{\infty}(V, \mathbb{R})$. 
(b) Let $(\Theta, f): \mathcal{S}_{\text {sld }}^{\infty}(U, \mathbb{R}) \rightarrow \mathcal{S}_{\text {sld }}^{\infty}(V, \mathbb{R})$ be a morphism of $\mathcal{S}$-sheafs. Then $(\Theta, f)$ induces a uniquely determined morphism of $\mathcal{S}$-sheaves $(\theta, f)$ : $\mathcal{S}_{u}^{\infty}(U, \mathbb{R}) \rightarrow \mathcal{S}_{u}^{\infty}(V, \mathbb{R})$.

Proof. Let $U^{\prime} \subset U$, then $\theta^{\prime}(h)=h \circ f$. Define $\Theta^{\prime}: \mathcal{S}_{\text {sld }}^{\infty}(U, \mathbb{R}) \rightarrow \mathcal{S}_{\text {sld }}^{\infty}(V, \mathbb{R})$ by $\Theta^{\prime}(h)=h \circ f$. So $\Theta^{\prime}$ is an $\mathbb{R}$-algebra homomorphism. Since $\theta^{\prime}$ maps strictly positive functions to strictly positive functions, $\Theta^{\prime}$ is the unique homomorphism. The second statement follows from the fact that $f$ is always a uniform $\mathcal{S}$ function.

\section{REFERENCES}

[1] E. Bierstone and P. D. Milman, Semianalytic and subanalytic sets, Inst. Hautes Études Sci. Publ. Math. 67 (1988), 5-42. MR 0972342

[2] F. Blais, R. Moussu and J.-P. Rolin, Non-oscillating integral curves and o-minimal structures, Analyzable functions and applications, Contemp. Math., vol. 373, Amer. Math. Soc., Providence, RI, 2005, pp. 103-112. MR 2130827

[3] J. Bochnak, M. Coste and M.-F. Roy, Real algebraic geometry, Ergebnisse der Mathematik und ihrer Grenzgebiete (3), vol. 36, Springer, Berlin, 1998. MR 1659509

[4] M. Coste, An introduction to o-minimal geometry, Dottorato di Ricerca in Mathematica, Dip. Mat. Univ. Pisa, Instituti Editoriali e Poligrafici Internazionali, 2000.

[5] J. Denef and L. van den Dries, p-adic and real subanalytic sets, Ann. of Math. (2) 128 (1988), no. 1, 79-138. MR 0951508

[6] L. van den Dries, Tame topology and o-minimal structures, London Math. Soc. Lecture Note Series, vol. 248, Cambridge University Press, Cambridge, 1998. MR 1633348

[7] L. van den Dries, A generalization of the Tarski-Seidenberg theorem, and some nondefinability results, Bull. Amer. Math. Soc. (N.S.) 15 (1986), no. 2, 189-193. MR 0854552

[8] L. van den Dries, A. Macintyre and D. Marker, The elementary theory of restricted analytic fields with exponentiation, Ann. of Math. (2) 140 (1994), no. 1, 183205. MR 1289495

[9] L. van den Dries and C. Miller, Geometric categories and o-minimal structures, Duke Math. J. 84 (1996), no. 2, 497-540. MR 1404337

[10] L. van den Dries and P. Speissegger, The field of reals with multisummable series and the exponential function, Proc. Lond. Math. Soc. (3) 81 (2000), no. 3, 513565. MR 1781147

[11] L. van den Dries and P. Speissegger, The real field with convergent generalized power series, Trans. Amer. Math. Soc. 350 (1998), no. 11, 4377-4421. MR 1458313

[12] G. A. Efroymson, The extension theorem for Nash functions, Real algebraic geometry and quadratic forms (Rennes, 1981), Lecture Notes in Math., vol. 959, Springer, Berlin, 1982, pp. 343-357. MR 0683141

[13] J. Escribano, Approximation theorems in o-minimal structures, Illinois J. Math. 46 (2002), no. 1, 111-128. MR 1936078

[14] A. Fischer, Smooth functions in o-minimal structures, Adv. Math. 218 (2008), no. 2, 496-514. MR 2407944

[15] A. Fischer, On compositions of subanalytic functions, preprint, 2008.

[16] A. Fischer, On sums of subanalytic functions, preprint, 2008.

[17] A. Grothedieck, Esquisse d'un programme, geometric Galois actions, London Math. Soc. Lecture Notes, vol. 242, Cambridge University Press, Cambridge, 1997, pp. 5-48.

[18] G. O. Jones, Local to global methods in o-minimal expansions of fields, Doctoral thesis, Wolfson College, University of Oxford, 2006. 
[19] T. Kaiser, J.-P. Rolin and P. Speissegger, Transition maps at non-resonant hyperbolic singularities are o-minimal, J. Reine Angew. Math. 637 (2009), 1-45. MR 2572245

[20] K. Kurdyka and W. Pawlucki, Subanalytic version of Whitney's extension theorem, Studia Math. 124 (1997), no. 3, 269-280. MR 1456425

[21] O. Le Gal and J.-P. Rolin, Une structure o-minimale sans décomposition cellulaire $C^{\infty}$, C. R. Math. Acad. Sci. Paris 346 (2008), 309-312. MR 2414175

[22] J.-M. Lion and P. Speissegger, Analytic stratification in the Pfaffian closure of an o-minimal structure, Duke Math. J. 103 (2000), no. 2, 215-231. MR 1760626

[23] C. Miller, Infinite differentiability in polynomially bounded o-minimal structures, Proc. Amer. Math. Soc. 123 (1995), no. 8, 2551-2555. MR 1257118

[24] C. Miller, Exponentiation is hard to avoid, Proc. Amer. Math. Soc. 122 (1994), no. 1, 257-259. MR 1195484

[25] R. Palais, Equivariant real algebraic digerential topology, Part I: Smoothness categories and Nash manifolds, notes, Brandeis University, 1972.

[26] D. Pecker, On Efroymson's extension theorem for Nash functions, J. Pure Appl. Algebra 37 (1985), no. 2, 193-203. MR 0796409

[27] J.-P. Rolin, F. Sanz and R. Schäfke, Quasi-analytic solutions of analytic ordinary differential equations and o-minimal structures, Proc. Lond. Math. Soc. (3), 95 (2007), 413-442. MR 2352566

[28] J.-P. Rolin, P. Speissegger and A. J. Wilkie, Quasianalytic Denjoy-Carleman classes and o-minimality, J. Amer. Math. Soc. 16 (2003), no. 4, 751-777. MR 1992825

[29] M. Shiota, Nash manifolds, Lecture Notes in Math., vol. 1269, Springer, Berlin, 1987. MR 0904479

[30] M. Shiota, Approximation theorems for Nash mappings and Nash manifolds, Trans. Amer. Math. Soc. 293 (1986), no. 1, 319-337. MR 0814925

[31] M. Shiota, Abstract Nash manifolds, Proc. Amer. Math. Soc. 96 (1986), no. 1, 155162. MR 0813829

[32] M. Shiota, Geometry of subanalytic and semialgebraic sets, Birkhäuser, Boston, MA, 1997. MR 1463945

[33] M. Shiota, Classification of Nash manifolds, Ann. Inst. Fourier (Grenoble) 33 (1983), 209-232. MR 0723954

[34] P. Speissegger, The Pfaffian closure of an o-minimal structure, J. Reine Angew. Math. $\mathbf{5 0 8}$ (1999), 189-211. MR 1676876

[35] H. Whitney, Differentiable manifolds, Ann. of Math. (2) 37 (1936), no. 3, 645680. MR 1503303

[36] A. J. Wilkie, Model completeness results for expansions of the ordered field of real numbers by restricted Pfaffian functions and the exponential function, J. Amer. Math. Soc. 9 (1996), 1051-1094. MR 1398816

[37] A. J. Wilkie, A theorem of the complement and some new o-minimal structures, Selecta Math. (N.S.) 5 (1999), no. 4, 397-421. MR 1740677

[38] A. J. Wilkie, On defining $C^{\infty}$, J. Symbolic Logic 59 (1994), no. 1, 344. MR 1264984

Andreas Fischer, Comenius Gymnasium Datteln, Suedring 150, 45711 Datteln, GERMANY

E-mail address: el.fischerandreas@live.de 